\section{Prevalência e fatores associados à desnutrição e ao excesso de peso em menores de cinco anos nos seis maiores municípios do Maranhão}

\author{
Prevalence and factors associated \\ to malnutrition and excess weight \\ among under five year-olds in the six \\ largest cities of Maranhão
}

\section{Deysianne Costa das Chagas}

Antônio Augusto Moura da Silva

Rosangela Fernandes Lucena Batista

Vanda Maria Ferreira Simões

Zeni Carvalho Lamy

Liberata Campos Coimbra

\section{Maria Teresa Seabra Soares de Britto e Alves}

Programa de Pós Graduação em Saúde Coletiva da Universidade Federal do Maranhão - UFMA.

Fontes de financiamento: Coordenação de Aperfeiçoamento de Pessoal de Nível Superior CAPES pela bolsa de mestrado concedida à Deysianne Costa das Chagas (Código 20001010015M4) e Secretaria de Estado da Saúde do Maranhão/Fundação de Amparo à Pesquisa do Maranhão FAPEMA, pelo suporte financeiro (Processo $n^{\circ} 0192.00 / 04$ ).

Correspondência: Deysianne Costa das Chagas. Rua Santo Adalberto, 04 - Residencial Granada

Bairro Forquilha, São Luis, MA CEP 65054-115. E-mail: deysiannechagas@yahoo.com.br

\section{Resumo}

Prevalências de desnutrição e excesso de peso em menores de cinco anos e sua associação com fatores socioeconômicos, sanitários e demográficos foram estimadas nos seis maiores municípios do Maranhão, em 2006/2007. Por meio de inquérito domiciliar por amostragem 1.214 crianças menores de cinco anos foram aleatoriamente selecionadas. Foi utilizada amostragem por conglomerados em dois estágios, representativa dos seis municípios maranhenses com mais de cem mil habitantes. Foram aplicados questionários padronizados para as mães ou responsáveis pelas crianças e aferidos peso e estatura. Para classificação da desnutrição foram utilizados os pontos de corte $<-2$ escores z pelos indicadores peso para idade, peso para estatura e estatura para idade. Para a classificação do excesso de peso foram considerados $>+2$ escores $\mathrm{z}$, de acordo com o indicador peso para estatura, seguindo recomendações da Organização Mundial da Saúde. Pelo índice peso para idade a prevalência de desnutrição foi de $4,5 \%$, pelo índice estatura para idade 8,5\% estavam com desnutrição pregressa e pelo índice peso para estatura $3,9 \%$ encontravam-se com desnutrição atual, enquanto 6,7\% apresentavam excesso de peso. Crianças de famílias chefiadas por mulheres apresentaram menores prevalências de desnutrição (Razão de Prevalências $=0,4$ ). Variáveis socioeconômicas não estiveram associadas à desnutrição ou ao excesso de peso. Recebimento de benefício do programa bolsa família não foi associado à desnutrição ou excesso de peso. A prevalência de desnutrição infantil foi baixa, mas o excesso de peso foi mais prevalente do que a desnutrição. Não foi detectada desigualdade social em relação à desnutrição em crianças menores de cinco anos, sugerindo evolução favorável no sentido de maior equidade.

Palavras-chave: Desnutrição infantil. Sobrepeso infantil. Transtornos nutricionais. Saúde infantil. 


\section{Abstract}

Prevalences of malnutrition and overweight among children under five years and its association with socioeconomic, demographic and health indicators were estimated for the six largest municipalities of Maranhão, in 2006/2007. By means of a household survey, a sample of 1214 children under five years of age was randomly selected. Two-stage cluster sampling was used, representing the six municipalities of Maranhão with over one hundred thousand inhabitants. Standardized questionnaire was administered to mothers or guardians and trained personnel measured weight and height or length. For classification of malnutrition cutoff points of $<-2 \mathrm{z}$ scores for weight-for-age, weight-for-length/height and length/ height-for-age were used. Overweight was considered when weight for heithg was $>+2$ z score, following World Health Organization guidelines. By weight-for-age malnutrition prevalence was 4.5 , by length/height-for-age $8.5 \%$ were stunted and by the weight-for-length/height $3.9 \%$ were malnourished (wasting), while $6.7 \%$ were overweight. Children of families headed by women had lower prevalence of malnutrition (prevalence ratio $=0.4$ ). Socioeconomic variables were not associated with malnutrition or overweight. Participation in money transfer programs from the government was not associated with malnutrition or overweight. The prevalence of malnutrition was low, but being overweight was more prevalent than malnutrition. Social inequality was not detected in relation to malnutrition in children under five years of age, suggesting a favorable trend towards greater equity.

Keywords: Child malnutrition. Children overweight. Children's nutrition disorders. Infant health.

\section{Introdução}

A desnutrição é caracterizada como uma condição patológica decorrente da falta de energia e proteínas, em variadas proporções, podendo ser agravada por infecções repetidas ${ }^{1}$. Já a obesidade, tanto infantil como nos adultos, é definida por um acúmulo excessivo de massa de gordura ${ }^{2}$.

Há evidências exaustivas de que déficits de crescimento na infância estão associados à maior mortalidade, doenças infecciosas, prejuízo para o desenvolvimento psicomotor, menor aproveitamento escolar e diminuição da altura e da capacidade produtiva na idade adulta ${ }^{3,4}$. No caso do sexo feminino, o retardo do crescimento na infância está associado à baixa estatura e a um maior risco de gerar crianças com baixo peso ao nascer ${ }^{5}$, o que demonstra o efeito intergeracional da desnutrição.

Estudo realizado por Monteiro (2009) ${ }^{6}$ evidenciou redução da desnutrição infantil no Brasil de cerca de 50\% de 1996 a 2006/7. Em contraponto ao declínio da desnutrição, tem sido observado aumento nas prevalências de sobrepeso e obesidade infantis. Atualmente, o aumento desses índices tem se tornado um problema de saúde pública, já que a obesidade acarreta prejuízos à saúde dos indivíduos, tais como a síndrome metabólica, risco elevado de desenvolvimento de hipertensão, dislipidemia e diabetes mellitus tipo 2, osteoartrite e alguns tipos de cânceres? ${ }^{7}$. Sua prevalência tem aumentado de forma rápida e está associada a mudanças nos hábitos de vida, dentre os quais citamos o aumento do sedentarismo e hábitos alimentares inadequados.

No Brasil, o déficit nutricional está sendo reduzido, enquanto aumenta o excesso de peso $^{8}$. Nos últimos anos verificou-se que a prevalência de excesso de peso para estatura em crianças menores de 5 anos já ultrapassava a prevalência de déficit de peso para estatura em todas as regiões do país. A Pesquisa Nacional sobre Demografia e Saúde-PNDS-de 2006 revelou que 7,3\% de crianças menores de 5 anos apresentavam 
excesso de peso para estatura e 1,5\% tinham déficit de peso para estatura9 ${ }^{9}$

Em estudo realizado em 1996 no Maranhão foi estimada prevalência elevada de desnutrição infantil em menores de cinco anos, $11,9 \%$ pelo indicador peso para idade, em $1996^{10}$ superior à estimada para o Nordeste, 6,3\% para o mesmo indicador no mesmo ano ${ }^{9}$. Entretanto, os estudos até então disponíveis de base populacional não estimaram a prevalência de excesso de peso em menores de 5 anos no Maranhão.

A implantação das atuais políticas sociais, dentre as quais podemos citar os programas de transferência de renda, tem desempenhado importante papel na diminuição das iniquidades sociais, em relação à desnutriçãa $0^{6}$ e à mortalidade infantil ${ }^{11}$. Entretanto, poucos estudos têm sido realizados para verificar se há diferenças nas prevalências de desnutrição e excesso de peso, comparando-se aqueles que recebem bolsa família com os demais grupos.

A desnutrição tem sido associada à pobreza, baixa escolaridade materna, precárias condições de moradia e saneamento, maior número de moradores na casa, assim como com idade materna inferior a $20 \operatorname{anos}^{6,12}$.

Atualmente, não se conhecem as prevalências de desnutrição e excesso de peso e nem os fatores associados a esses agravos em crianças maranhenses. Também não se sabe em que magnitude a desigualdade social se associa com a desnutrição. É importante avaliar em regiões menos desenvolvidas se a desnutrição está diminuindo de importância e se o excesso de peso já surge como problema de saúde pública. Desta forma, o objetivo desse estudo foi avaliar as prevalências e os fatores associados à desnutrição e ao excesso de peso em menores de cinco anos nos seis municípios mais populosos do Estado do Maranhão, um dos mais pobres da Federação.

\section{Metodologia}

Foram utilizados dados do estudo transversal intitulado "Atenção à Saúde no
Maranhão", desenvolvido em 2006/7, em amostra probabilística, investigada por meio de inquérito domiciliar com crianças menores de cinco anos, nos seis municípios com mais de 100 mil habitantes do Estado do Maranhão (São Luis, Imperatriz, Caxias, Codó, São José de Ribamar e Timon) ${ }^{13}$. Esse estudo teve o objetivo de estudar a prevalência dos principais indicadores de saúde e a utilização de serviços pela população residente nesses seis municípios mais populosos do Estado.

Foi utilizado um processo de amostragem por conglomerados com probabilidade proporcional à população de cada município, em duas etapas. Na primeira foram selecionados 100 setores censitários dos seis municípios, com probabilidade proporcional à população a partir da base censitária de 2000. Na segunda foi sorteado o quarteirão inicial dentro de cada setor, sendo em seguida sorteado um ponto inicial dentro de cada quarteirão, que foi percorrido até que se entrevistassem a mãe ou o responsável de 12 crianças menores de cinco anos, em cada setor censitário, não se limitando o número de menores de cinco anos incluídos por domicílio. No segundo estágio, a unidade de amostragem foi o indivíduo, mas o processo de amostragem foi realizado em nível de domicílio. Em casos em que esse número de crianças não fosse encontrado no quarteirão, era feito um sorteio de outro quarteirão dentro do mesmo setor até atingir o número desejado. Se na última casa houvesse mais crianças do que o necessário para se completar 12 pessoas por setor, todas as crianças eram incluídas e, nestes casos, o número de crianças amostradas por setor ultrapassou 12. Como os domicílios foram selecionados com aproximadamente a mesma probabilidade, não houve necessidade de se realizar ponderação.

Para o cálculo do tamanho amostral assumiu-se uma prevalência de $5 \%$, tanto para desnutrição como para excesso de peso, e uma diferença de prevalência entre grupo exposto e não exposto de $5 \%$, poder do teste de 80 , probabilidade de erro tipo I de 0,05 , resultando num total de 918 crianças. 
Para compensar o uso de delineamento complexo de amostragem, a amostra final foi composta por 1.214 crianças.

Foram coletadas as medidas antropométricas das crianças, como peso $(\mathrm{kg}) \mathrm{e}$ estatura $(\mathrm{cm})$. $\mathrm{O}$ peso foi medido em quilogramas e aferido em balanças digitais da marca Plenna ${ }^{\circledR}$ com capacidade de $150 \mathrm{~kg}$ e graduação de $100 \mathrm{~g}$, posicionada em local plano. A criança foi pesada sem roupa e no colo da mãe ou responsável, e o seu peso definido pela diferença obtida. O comprimento foi aferido para crianças de até dois anos e a altura medida nas demais. Para medida do comprimento foi utilizado infantômetro da marca Alturexata ${ }^{\circledR}$ com graduação de 0,1 $\mathrm{cm}$, com a criança em posição de decúbito dorsal, pernas relaxadas, cabeça apoiada enquanto a peça móvel do infantômetro era deslocada até tocar os pés alinhados da criança. A altura foi medida com a criança em posição ortostática, descalça, com as pernas e calcanhares juntos, os braços ao longo do corpo e com a cabeça mantida no plano de Frankfurt. Para medida da altura foi utilizado estadiômetro da marca Alturexata ${ }^{\circledR}$ com graduação de $0,1 \mathrm{~cm}$.

A idade das crianças foi determinada com base na diferença entre a data de nascimento e a data da coleta de dados. Para a definição da classe econômica, utilizou-se a classificação proposta pela Associação Brasileira de Empresas de Pesquisa (ABEP) ${ }^{14}$.

Foram medidos os índices antropométricos estatura para idade (E/I), peso para idade $(\mathrm{P} / \mathrm{I})$ e peso para estatura $(\mathrm{P} / \mathrm{E})$ em valores de escore-Z (unidades de desvio-padrão), comparados ao padrão de referência da Organização Mundial da Saúde (OMS) ${ }^{15}$. Para classificação da desnutrição foram utilizados os pontos de corte $<-2$ escore-Ze para a classificação do excesso de peso pelo índice peso para estatura foram utilizados os pontos de corte $>+2$ escore-Z, segundo a classificação do Sistema de Vigilância Nutricional (SISVAN) ${ }^{16}$. Os índices foram calculados no programaWHO Antro 3.0.1 ${ }^{17}$.

A variável "condições de moradia e saneamento" foi criada para sumarizar variáveis referentes a essas condições por meio de um escore. Para a construção deste escore foram consideradas as variáveis tipo de moradia, abastecimento de água, destino dos dejetos e lixo. Cada uma dessas variáveis foi codificada em adequada (0) e inadequada (1). Os valores foram somados, obtendo-se o valor do escore. Portanto, quanto menor foi o valor do escore, melhores eram as condições de moradia e saneamento. As demais variáveis foram obtidas a partir de questionário padronizado aplicado por pesquisadores treinados em entrevista com a mãe ou responsável pela criança.

A variável dependente utilizada no modelo estatístico foi o índice peso para estatura, dividido em três categorias: eutrofia (utilizada como categoria de referência), desnutrição e excesso de peso. A análise estatística foi realizada no pacote Stata, versão $9,0^{18}$, utilizando-se o conjunto de comandos svy. O uso de estimadores convencionais poderia subestimar consideravelmente a variância das estimativas pontuais ${ }^{18}$. O conjunto de comandos svy do Stata levou em consideração o delineamento amostral complexo (amostragem por conglomerados em dois estágios e estratificação prévia por município) ${ }^{17}$.

Inicialmente foi realizada a análise descritiva e posteriormente foi feita a análise univariável, utilizando-se a regressão logística multinomial simples, com estimativa da razão de prevalências não ajustada e intervalo de confiança de 95\% (IC95\%). A análise multivariável foi realizada conforme o modelo hierárquico definido a priore (Figura 1). O primeiro bloco (nível hierárquico 1) incluiu as variáveis socioeconômicas: classe econômica, escolaridade do chefe da família; o segundo bloco - Raça/etnia - incluiu a variável cor da pele do chefe de familia; o terceiro bloco foi composto por variáveis ambientais medidas por escore, resumindo as condições de moradia e saneamento; o quarto bloco incluiu programas governamentais: recebimento do benefício Bolsa Família e acompanhamento pelo Programa Saúde da Família (PSF); o quinto bloco foi composto por variáveis demográficas: idade materna, número de moradores na casa 
e sexo do chefe da família; o sexto bloco incluiu variáveis biológicas: sexo e idade da criança.

No primeiro bloco (nível hierárquico 1) foram incluídas as variáveis socioeconômicas, mesmo aquelas não significativas na análise bivariável. As variáveis significativas nesta análise ( $p$-valor $<0,10$ ) foram conservadas no modelo e entraram no ajuste do próximo bloco, no caso a raça/etnia (nível hierárquico 2). Para os blocos seguintes do modelo, o mesmo procedimento foi empregado. Ao final do procedimento acima, foram criados dois modelos finais de fatores associados: um para o déficit e outro para o excesso de peso para estatura.

Foi também realizada analise hierarquizada para o déficit estatura para idade, utilizando-se modelo de regressão logística.

Os protocolos do estudo foram aprovados pelo Comitê de Ética em Pesquisa da Universidade Federal do Maranhão em parecer consubstanciado no. №275/06 e seguiram as normas da resolução 196/96 do Conselho Nacional de Saúde e suas complementares sobre pesquisa envolvendo seres humanos.

\section{Resultados}

Pelo critério peso para idade, a prevalência de desnutrição foi de $4,5 \%$. Pelo critério peso para estatura, 3,9\% apresentaram desnutrição e 6,7\% excesso de peso. Baixa estatura para idade foi observada em $8,5 \%$ dos menores de cinco anos (Tabela 1).

$\mathrm{Na}$ análise univariável crianças cujas famílias eram chefiadas por mulheres apresentaram menor prevalência de desnutrição ( $\mathrm{RP}=0,4$, IC 95\% 0,2-0,8). As prevalências de desnutrição foram semelhantes para os que recebiam ou não benefício de transferência de renda do governo (Tabela 2).

As Tabelas 3 e 4 mostram os resultados da análise multivariável. Mesmo controlando o efeito das variáveis socioeconômicas, ambientais e biológicas, estas não apresentaram associação estatisticamente significante com a desnutrição nem com o excesso de peso. Das variáveis estudadas,

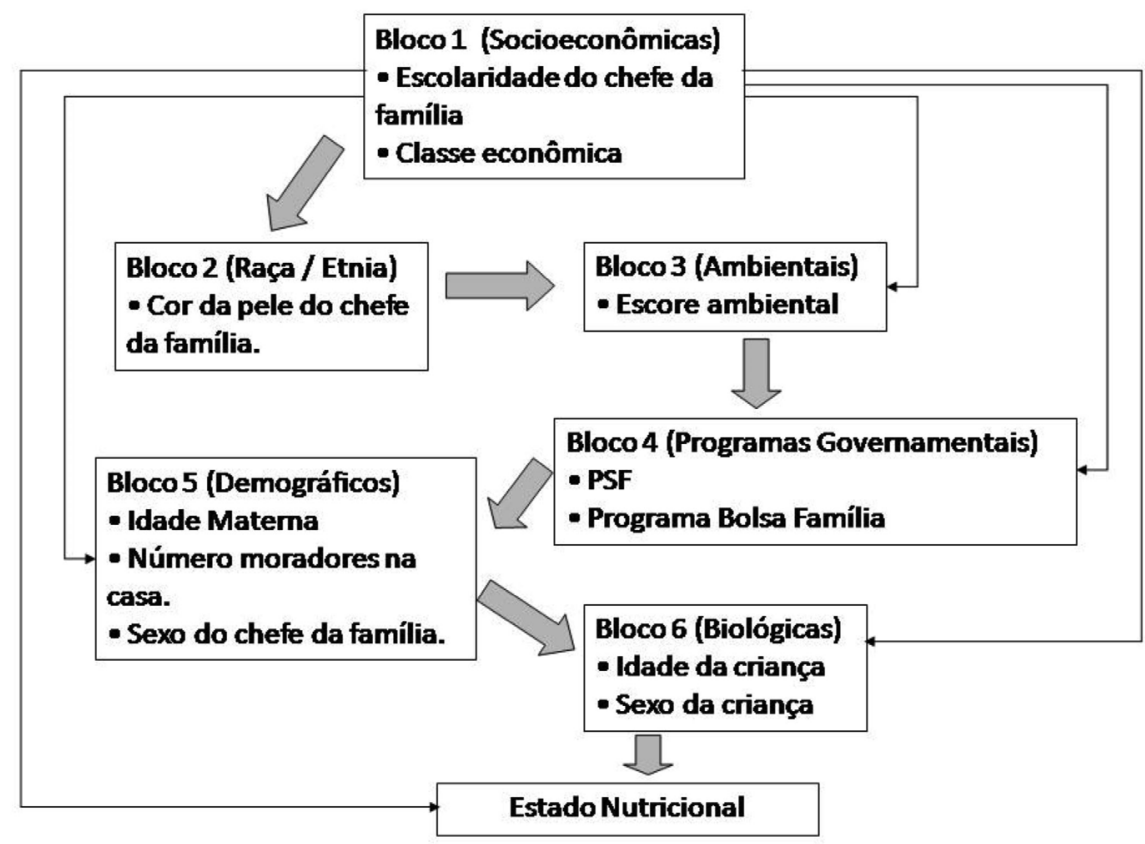

Figura 1 - Modelo teórico para investigação de fatores determinantes do estado nutricional de menores de cinco anos, estruturado em blocos hierarquizados.

Figure 1 - Theoretical model used to investigate the determinant factors of nutritional status in children under five years, structured in hierarchical groups. 
Tabela 1 - Estado nutricional segundo os critérios peso para idade*, peso para estatura** e estatura para idade* em menores de cinco anos nos seis municípios mais populosos do Maranhão, 2006/2007.

Table 1 - Nutritional status according to the criteria weight for age*, weight-for-length/height** and length/height-for-age* among under five year old children in the six most populous cities from Maranhão state, 2006/2007.

\begin{tabular}{lccc}
\hline Indicadores & $\mathrm{n}$ & $\%$ & IC $95 \%$ \\
\hline Critério Peso para idade & & & \\
$\quad$ Peso Muito Baixo para a idade & 14 & 1,2 & $0,6-1,8$ \\
$\quad$ Peso Baixo para a idade & 39 & 3,3 & $2,2-4,3$ \\
$\quad$ Peso Adequado ou Eutrófico & 1146 & 95,6 & $94,4-96,7$ \\
Critério Peso para estatura & & & \\
$\quad$ Peso Baixo para a estatura & 46 & 3,9 & $2,8-5,0$ \\
$\quad$ Peso Adequado ou Eutrófico & 1051 & 89,4 & $87,6-91,1$ \\
$\quad$ Peso Elevado para a estatura & 79 & 6,7 & $5,3-8,1$ \\
Critério Estatura para idade & & & \\
$\quad$ Baixa Estatura para a idade & 100 & 8,5 & $6,9-10,1$ \\
$\quad$ Estatura Adequada para a idade & 1076 & 91,5 & $89,9-93,1$ \\
\hline
\end{tabular}

*WHO, 2006; 2007. **WHO, 2006

após o ajuste, apenas chefe da família do sexo feminino permaneceu independentemente associada à desnutrição (Razão de prevalências $=0,4$ ).

$\mathrm{Na}$ análise de regressão logística hierarquizada nenhum fator foi associado ao déficit de estatura para idade (dados não mostrados em tabela).

\section{Discussão}

As prevalências estimadas de desnutrição e excesso de peso foram baixas. A prevalência de excesso de peso superou a de desnutrição, considerando-se o índice peso para estatura em menores de cinco anos residentes nos seis municípios mais populosos do Maranhão. Os fatores socioeconômicos não se associaram ao estado nutricional das crianças, e famílias chefiadas por mulheres apresentaram menor prevalência de desnutrição pelo critério peso para estatura. Receber benefício de programas de transferência de renda não esteve associado à desnutrição ou ao excesso de peso.

Em artigo publicado por Martorell 1998 ${ }^{19}$, as prevalências de baixo peso para estatura foram de 1,2\% na República Dominicana,
$1,1 \%$ em Honduras e Peru e $0,4 \%$ nos Estados Unidos, valores menores do que o deste estudo. Em relação ao excesso de peso para estatura, as prevalências encontradas foram de 4,6\% na República Dominicana, 1,4\% em Honduras, $4,7 \%$ no Peru e 7,4\% nos Estados Unidos, valores também inferiores ao obtido neste estudo, exceto nos Estados Unidos, onde a prevalência foi superior.

Segundo dados da PNDS em 2006, o déficit de peso para estatura no Brasil foi de $1,5 \%$, valor inferior aos $3,9 \%$ encontrados nesta pesquisa nos seis municípios maranhenses pesquisados, indicando que a prevalência de desnutrição atual é mais que o dobro daquela observada no Brasil. Em relação ao déficit de estatura para idade, a prevalência obtida na mesma pesquisa foi de 6,7\%, valor também inferior aos 8,5\% encontrados neste estudo ${ }^{9}$. Esses resultados mostram que a prevalência de desnutrição pregressa nesses municípios foi baixa, mas ainda superior à nacional.

Embora as prevalências de desnutrição neste estudo sejam superiores às nacionais, estudos realizados anteriormente no Maranhão, mostravam índices mais elevados que os atuais. Quando comparados aos 
Tabela 2 - Análise não ajustada por regressão logística multinomial dos fatores associados à desnutrição e ao excesso de peso pelo critério peso para estatura em crianças menores de cinco anos nos seis municípios mais populosos do Estado do Maranhão, 2006-2007.

Table 2 - Non-adjusted analysis by multinomial logistic regression of the factors associated to underweight and excess weight according to the weight-for-length/height criterion among under five year-old children who live in the six most populous cities, Maranhão state, 2006/2007.

\begin{tabular}{|c|c|c|c|c|c|c|}
\hline \multirow[b]{2}{*}{ Variáveis } & \multicolumn{3}{|c|}{ Desnutrição } & \multicolumn{2}{|c|}{ Excesso de Peso } & \multirow[b]{2}{*}{ p-valor } \\
\hline & $\begin{array}{l}\text { Razão de } \\
\text { Prevalências }\end{array}$ & $\begin{array}{c}\text { Intervalo de } \\
\text { Confiança de } \\
95 \%\end{array}$ & $\mathrm{p}$-valor & $\begin{array}{l}\text { Razão de } \\
\text { Prevalências }\end{array}$ & $\begin{array}{c}\text { Intervalo de } \\
\text { Confiança de } \\
95 \%\end{array}$ & \\
\hline Sexo da criança & & & 0,47 & & & 0,21 \\
\hline Masculino & \multicolumn{2}{|c|}{ Referência } & & \multicolumn{2}{|c|}{ Referência } & \\
\hline Feminino & 0,8 & $0,5-1,4$ & & 1,3 & $0,9-2,1$ & \\
\hline Idade da criança (anos) & & & 0,72 & & & 0,52 \\
\hline$\leq 2$ & \multicolumn{2}{|c|}{ Referência } & & \multicolumn{2}{|c|}{ Referência } & \\
\hline$>2$ & 0,9 & $0,5-1,7$ & & 1,1 & $0,8-1,7$ & \\
\hline Cor da pele & & & 0,52 & & & 0,66 \\
\hline Não preta & \multicolumn{2}{|c|}{ Referência } & & \multicolumn{2}{|c|}{ Referência } & \\
\hline Preta & 1,2 & $0,6-2,5$ & & 0,9 & $0,6-1,5$ & \\
\hline Sexo do chefe de família & & & 0,01 & & & 0,60 \\
\hline Masculino & \multicolumn{2}{|c|}{ Referência } & & \multicolumn{2}{|c|}{ Referência } & \\
\hline Feminino & 0,4 & $0,2-0,8$ & & 1,11 & $0,7-1,9$ & \\
\hline Escolaridade do chefe da família (anos) & & & 0,83 & & & 0,60 \\
\hline$\leq 8$ & \multicolumn{2}{|c|}{ Referência } & & \multicolumn{2}{|c|}{ Referência } & \\
\hline$\geq 9$ & 0,9 & $0,5-1,7$ & & 1,1 & $0,7-1,8$ & \\
\hline Classe econômica & & & 0,75 & & & 0,46 \\
\hline$A-B-C$ & \multicolumn{2}{|c|}{ Referência } & & \multicolumn{2}{|c|}{ Referência } & \\
\hline$D-E$ & 1,1 & $0,6-2,2$ & & 1,2 & $0,7-2,0$ & \\
\hline Condições de moradia e saneamento & & & 0,77 & & & 0,87 \\
\hline Adequadas & \multicolumn{2}{|c|}{ Referência } & & \multicolumn{2}{|c|}{ Referência } & \\
\hline Inadequadas & 1,1 & $0,6-2,2$ & & 1,0 & $0,6-1,6$ & \\
\hline Recebimento do Bolsa Família & & & 0,45 & & & 0,94 \\
\hline Não & \multicolumn{2}{|c|}{ Referência } & & \multicolumn{2}{|c|}{ Referência } & \\
\hline Sim & 1,3 & $0,7-2,3$ & & 1,0 & $0,6-1,7$ & \\
\hline Idade materna (anos) & & & 0,70 & & & 0,68 \\
\hline$\leq 20$ & \multicolumn{2}{|c|}{ Referência } & & \multicolumn{2}{|c|}{ Referência } & \\
\hline$>20$ & 1,2 & $0,4-3,4$ & & 1,2 & $0,5-2,6$ & \\
\hline Número de pessoas na casa & & & 0,15 & & & 0,22 \\
\hline 1 a 5 & \multicolumn{2}{|c|}{ Referência } & & \multicolumn{2}{|c|}{ Referência } & \\
\hline$\geq 6$ & 1,6 & $0,9-2,9$ & & 0,7 & $0,4-1,2$ & \\
\hline
\end{tabular}

resultados deste estudo, observou-se queda na prevalência de desnutrição pregressa, que passou de 30,6\% em 1991, para 24,0\% em $1996^{10}$, em amostra representativa de crianças menores de cinco anos. Neste estudo a prevalência de desnutrição pregressa foi de 8,5\% em 2006/7 nos seis municípios mais populosos do Estado, valor inferior ao de 1996 obtido para o Estado como um todo, o que sugere que a desnutrição pregressa diminuiu no período.

Post $^{20}$, estudando crianças com um ano de idade de duas coortes de base populacional no sul do Brasil, verificou que o excesso de peso apresentou uma tendência crescente, apresentando pelo índice peso para estatura prevalência de 4,0\% em 1982 e $6,7 \%$ em 1993. A prevalência de excesso 
Tabela 3 - Análise hierarquizada ajustada por regressão logística multinomial dos fatores associados à desnutrição pelo critério peso para estatura em crianças menores de cinco anos nos seis municípios mais populosos do Estado do Maranhão, 2006-2007.

Table 3 - Adjusted hierarchical analysis by multinomial logistic regression of the factors associated to underweight according to the weight-for-length/height criterion among under five year-old children who live in the six most populous cities, Maranhão state, 2006/2007.

\begin{tabular}{|c|c|c|c|c|c|c|c|c|c|c|c|c|c|}
\hline \multirow{2}{*}{ Variáveis } & & \multicolumn{2}{|l|}{ Bloco 1} & \multicolumn{2}{|l|}{ Bloco 2} & \multicolumn{2}{|l|}{ Bloco 3} & \multicolumn{2}{|l|}{ Bloco 4} & \multicolumn{2}{|l|}{ Bloco 5} & \multicolumn{2}{|l|}{ Bloco 6} \\
\hline & & RP (IC 95\%) & $\mathrm{p}$ & RP (IC 95\%) & $\mathrm{p}$ & RP (IC 95\%) & $\mathrm{p}$ & RP (IC 95\%) & $\mathrm{p}$ & RP (IC 95\%) & $\mathrm{p}$ & RP (IC 95\%) & $p$ \\
\hline \multirow{3}{*}{$\begin{array}{l}\text { Classe } \\
\text { econômica }\end{array}$} & $A-B$ & 1 & & & & & & & & & & & \\
\hline & C & $2,9(0,7-13,2)$ & 0,16 & & & & & & & & & & \\
\hline & $D-E$ & $3,0(0,6-15,1)$ & 0,17 & & & & & & & & & & \\
\hline \multirow{2}{*}{$\begin{array}{l}\text { Escolaridade } \\
\text { do chefe de } \\
\text { família }\end{array}$} & $\leq 8$ anos & 1 & & & & & & & & & & & \\
\hline & $\geq 9$ anos & $1,2(0,6-2,3)$ & 0,69 & & & & & & & & & & \\
\hline \multirow{3}{*}{$\begin{array}{l}\text { Cor da pele } \\
\text { do chefe de } \\
\text { família }\end{array}$} & Branca & & & 1 & & & & & & & & & \\
\hline & Pardo & & & $1,4(0,6-3,3)$ & 0,46 & & & & & & & & \\
\hline & Preto & & & $1,2(0,3-4,4)$ & 0,76 & & & & & & & & \\
\hline \multirow{2}{*}{$\begin{array}{l}\text { Condições } \\
\text { ambientais }\end{array}$} & Adequadas & & & & & 1 & & & & & & & \\
\hline & Inadequadas & & & & & $1,1(0,6-2,2)$ & 0,77 & & & & & & \\
\hline \multirow{2}{*}{$\begin{array}{l}\text { Recebimento } \\
\text { de Bolsa } \\
\text { família }\end{array}$} & Não & & & & & & & 1 & & & & & \\
\hline & Sim & & & & & & & $1,4(0,7-2,6)$ & 0,30 & & & & \\
\hline \multirow{2}{*}{$\begin{array}{l}\text { Acompanha } \\
\text { mento pelo } \\
\text { PSF }\end{array}$} & Não & & & & & & & 1 & & & & & \\
\hline & Sim & & & & & & & $0,9(0,4-1,7)$ & 0,70 & & & & \\
\hline \multirow{2}{*}{ Idade materna } & $<20$ anos & & & & & & & & & 1 & & & \\
\hline & $\geq 20$ anos & & & & & & & & & $1,1(0,4-3,3)$ & 0,82 & & \\
\hline \multirow{2}{*}{$\begin{array}{l}\text { Número de } \\
\text { pessoas na } \\
\text { casa }\end{array}$} & 5 ou menos & & & & & & & & & 1 & & & \\
\hline & 6 ou mais & & & & & & & & & $1,6(0,9-3,0)$ & 0,12 & & \\
\hline \multirow{2}{*}{$\begin{array}{l}\text { Sexo do chefe } \\
\text { de família }\end{array}$} & Masculino & & & & & & & & & 1 & & 1 & \\
\hline & Feminino & & & & & & & & & $0,4(0,2-0,8)$ & 0,01 & $0,4(0,2-0,8)$ & 0,01 \\
\hline \multirow{2}{*}{$\begin{array}{l}\text { Idade da } \\
\text { criança }\end{array}$} & $\leq 2$ anos & & & & & & & & & & & 1 & \\
\hline & $>2$ anos & & & & & & & & & & & $0,9(0,4-1,7)$ & 0,71 \\
\hline \multirow{2}{*}{$\begin{array}{l}\text { Sexo da } \\
\text { criança }\end{array}$} & Masculino & & & & & & & & & & & 1 & \\
\hline & Feminino & & & & & & & & & & & $0,8(0,4-1,3)$ & 0,34 \\
\hline
\end{tabular}

* RP=Razão de Prevalências, IC=Intervalo de Confiança; PSF=Programa de Saúde da Família

*PR: Prevalence Ratios, Cl: Confidence Interval, PSF: Programa de Saúde da Família (Family Health Program)

de peso neste estudo, segundo o mesmo índice, foi de 6,7\%, bem semelhante à observada em Pelotas, sul do Brasil, em 199320. Quando foram comparadas as taxas atuais de excesso de peso calculadas para os seis municípios mais populosos do Maranhão com estudos realizados em outros estados, observou-se que a prevalência de $6,7 \%$ de excesso de peso pelo índice peso para estatura obtida neste trabalho foi semelhante à verificada em Florianópolis, SC (6,8\%), e inferior à encontrada em São Leopoldo, RS $(9,8 \%)$ em crianças da mesma faixa etária $^{21,22}$. Achados como estes mostram que as diferenças nutricionais, antes evidentes entre crianças das regiões Nordeste e do Sul, já não são mais tão evidentes, mostrando-se mais homogêneas em todo o país.

Analisando estes resultados em comparação com os trabalhos anteriores, realizados em outros Estados, sugere-se que houve uma mudança no estado nutricional dessas crianças, com a diminuição da desnutrição e aumento do excesso de peso. Esse fato, porém, pode apontar uma evidência de que o processo de transição nutricional esteja se dando nessa faixa etária, onde as carências globais da desnutrição estão diminuindo e 
Tabela 4 - Análise hierarquizada ajustada por regressão logística multinomial dos fatores associados ao excesso de peso pelo critério peso para estatura em crianças menores de cinco anos nos seis municípios mais populosos do Estado do Maranhão, 2006-2007.

Table 4 - Adjusted hierarchical analysis by multinomial regression of the factors associated to excess weight according to the weight-for-length/height criterion among under five year-old children who live in the six most populous cities, Maranhão state, 2006/2007.

\begin{tabular}{|c|c|c|c|c|c|c|c|c|c|c|c|c|c|}
\hline \multirow{2}{*}{ Variáveis } & & \multicolumn{2}{|l|}{ Bloco 1} & \multicolumn{2}{|l|}{ Bloco 2} & \multicolumn{2}{|l|}{ Bloco 3} & \multicolumn{2}{|l|}{ Bloco 4} & \multicolumn{2}{|l|}{ Bloco 5} & \multicolumn{2}{|l|}{ Bloco 6} \\
\hline & & RP (IC 95\%) & $p$ & RP (IC 95\%) & $p$ & RP (IC 95\%) & $p$ & RP (IC $95 \%)$ & $p$ & RP (IC 95\%) & $\mathrm{p}$ & RP (IC 95\%) & $p$ \\
\hline \multirow{3}{*}{$\begin{array}{l}\text { Classe } \\
\text { econômica }\end{array}$} & $A-B$ & 1 & & & & & & & & & & & \\
\hline & C & $1,2(0,5-2,8)$ & 0,68 & & & & & & & & & & \\
\hline & $D-E$ & $1,8(0,7-4,3)$ & 0,21 & & & & & & & & & & \\
\hline \multirow{2}{*}{$\begin{array}{l}\text { Escolaridade } \\
\text { do chefe de } \\
\text { família }\end{array}$} & $\leq 8$ anos & 1 & & & & & & & & & & & \\
\hline & $\geq 9$ anos & $1,4(0,8-2,5)$ & 0,19 & & & & & & & & & & \\
\hline \multirow{3}{*}{$\begin{array}{l}\text { Cor da pele } \\
\text { do chefe de } \\
\text { família }\end{array}$} & Branca & & & 1 & & & & & & & & & \\
\hline & Parda & & & $1,0(0,6-1,9)$ & 0,88 & & & & & & & & \\
\hline & Preta & & & $0,9(0,4-2,4)$ & 0,91 & & & & & & & & \\
\hline \multirow{2}{*}{$\begin{array}{l}\text { Condições } \\
\text { ambientais }\end{array}$} & Adequadas & & & & & 1 & & & & & & & \\
\hline & Inadequadas & & & & & $1,0(0,6-1,6)$ & 0,87 & & & & & & \\
\hline \multirow{2}{*}{$\begin{array}{l}\text { Recebimento } \\
\text { de Bolsa } \\
\text { família } \\
\end{array}$} & Não & & & & & & & 1 & & & & & \\
\hline & Sim & & & & & & & $1,0(0,5-1,7)$ & 0,91 & & & & \\
\hline \multirow{2}{*}{$\begin{array}{l}\text { Acompanha } \\
\text { mento pelo } \\
\text { PSF } \\
\end{array}$} & Não & & & & & & & 1 & & & & & \\
\hline & Sim & & & & & & & $1,1(0,6-1,8)$ & 0,80 & & & & \\
\hline \multirow{2}{*}{ Idade materna } & $<20$ anos & & & & & & & & & 1 & & & \\
\hline & $\geq 20$ anos & & & & & & & & & $1,1(0,5-2,5)$ & 0,76 & & \\
\hline \multirow{2}{*}{$\begin{array}{l}\text { Número de } \\
\text { pessoas na } \\
\text { casa }\end{array}$} & 5 ou menos & & & & & & & & & 1 & & & \\
\hline & 6 ou mais & & & & & & & & & $0,7(0,4-1,2)$ & 0,17 & & \\
\hline \multirow{2}{*}{$\begin{array}{l}\text { Sexo do chefe } \\
\text { de família }\end{array}$} & Masculino & & & & & & & & & 1 & & 1 & \\
\hline & Feminino & & & & & & & & & $1,2(0,7-2,0)$ & 0,52 & $1,1(0,7-1,9)$ & 0,63 \\
\hline \multirow{2}{*}{$\begin{array}{l}\text { Idade da } \\
\text { criança }\end{array}$} & $\leq 2$ anos & & & & & & & & & & & 1 & \\
\hline & $>2$ anos & & & & & & & & & & & $1,1(0,7-1,7)$ & 0,60 \\
\hline \multirow{2}{*}{$\begin{array}{l}\text { Sexo da } \\
\text { criança }\end{array}$} & Masculino & & & & & & & & & & & 1 & \\
\hline & Feminino & & & & & & & & & & & $1,3(0,8-2,0)$ & 0,26 \\
\hline
\end{tabular}

o excesso de peso está aumentando, sem que haja substituição de uma pela outra. Segundo Batista Filho (2009) ${ }^{23}$, esse padrão tem se configurado como grande desafio para os países em desenvolvimento.

Muitos estudos associaram baixa renda à desnutrição, e alta renda ao excesso de peso $^{22,24}$. Entretanto, outros estudos têm demonstrado que as condições socioeconômicas das famílias não estão mais associadas ao estado nutricional infantil, como foi observado por Corso (2003) ${ }^{21}$. Em nosso estudo não foram verificadas associações estatisticamente significantes entre excesso de peso e desnutrição com escolaridade do chefe de família, classe econômica e outras variáveis socioeconômicas. Pode-se sugerir, a partir dos resultados encontrados, que as desigualdades estão sendo reduzidas no Maranhão, já que os fatores socioeconômicos não se associaram aos distúrbios nutricionais das crianças.

Em artigo publicado por Lima (2010) ${ }^{25}$ demonstrou-se que a evolução simultânea do poder aquisitivo familiar, da escolaridade materna, da disponibilidade de serviços de saneamento e de assistência à saúde, $\mathrm{e}$ dos antecedentes reprodutivos das mães, 
justificaria pouco mais da metade do declínio na prevalência da desnutrição infantil na região Nordeste no decênio 1986-1996, e quase dois terços no decênio 1996-2006. Isto sugere que a melhoria destes indicadores contribuiu para reduzir ou eliminar as desigualdades socioeconômicas em relação à desnutrição.

Ser criança de família chefiada por mulher mostrou-se associado à menor prevalência de desnutrição (RP 0,4, IC 95\% $0,2-0,8)$. De acordo com Carvalhaes ${ }^{26}$, a capacidade materna de manejar e otimizar os diversos recursos necessários a um adequado cuidado infantil influenciaria o estado nutricional da criança. Isto pode explicar estes achados na medida em que as mães chefes de família teriam poder para controlar e alocar os recursos disponíveis de forma mais conveniente para garantir a saúde nutricional de seus filhos.

Neste estudo transversal não se pode concluir que as associações observadas sejam causais, embora se tenha sugerido possíveis fatores associados às condições nutricionais dessas crianças. Como ponto forte do estudo destaca-se a sua base populacional. Em menores de cinco anos, a prevalência da desnutrição foi baixa nos municípios com maior número de habitantes e o excesso de peso já surge como problema de saúde pública, o que constitui grande desafio para os serviços e políticas de saúde.

Outro ponto é que o excesso de peso já é mais prevalente que a desnutrição nesses municípios. Isso sugere que, no processo de transição nutricional infantil, a desnutrição, apesar da tendência de diminuição, não foi substituída pelo padrão onde prevalece o excesso de peso. Esta situação é semelhante à terceira fase descrita por Batista Filho ${ }^{27}$, na qual o processo de transição nutricional teria, em escala populacional, o predomínio do binômio sobrepeso/obesidade sobre a desnutrição.

Desta forma, o estado nutricional infantil precisa ser monitorado e devem ser desenvolvidas políticas públicas para educação nutricional que abordem estes distúrbios nutricionais e sejam voltadas tanto para famílias de baixa renda quanto para as de renda mais alta.

\section{Colaboradores}

D. C. Chagas contribuiu na concepção do artigo, na discussão dos resultados e redação do texto. A. A. M. Silva, L. C. Coimbra, R. F. L. Batista, V. M. F. Simões e Z. C. Lamy contribuíram na interpretação e discussão dos resultados e na revisão final do texto. $\mathrm{M}$. T. S. S. B. Alves contribuiu na interpretação e discussão dos resultados, redação e revisão final do texto.

\section{Referências}

1. Lacerda EMA, Accioly E, Faria IG, Costa VM. Práticas de Nutrição Pediátrica. São Paulo: Editora Atheneu; 2006.

2. Frelut ML, Navarro J. Obesity in the child. Presse Med 2000; 10: 572-7.

3. Black RE, Allen LH, Bhutta ZA, Caulfield LE, de Onis M, Ezzati M et al. Maternal and child undernutrition: global and regional exposures and health consequences. Lancet 2008; 371(9608): 243-60.

4. Victora CG, Adair L, Fall C, Hallal PC, Martorell R, Richter L et al. Maternal and child undernutrition: consequences for adult health and human capital. Lancet 2008; 371(9609): 340-57.

5. Kramer MS. Determinants of low birth weight: methodological assessment and meta-analysis. Bull Word Health Organ 1987; 65: 663-737.
6. Monteiro CA, Benicio MHDA, Konno SC, Silva ACFd, Lima ALLd, Conde WL. Causas do declínio da desnutrição infantil no Brasil, 1996-2007. Rev Saúde Pública 2009; 43: 35-43.

7. Mello ED, Luft VC, Meyer F. Obesidade infantil: como podemos ser eficazes? J Pediatr 2004; 80: 173-82.

8. Monteiro CA, Mondini L, Souza ALM, Popkin BM. The nutrition transition in Brazil. Eur J Clin Nutr 1995; 49: 105-13.

9. Brasil. Ministério da Saúde. Pesquisa Nacional de Demografia e Saúde da Criança e da Mulher - PNDS 2006: dimensões do processo reprodutivo e da saúde da criança. Ministério da Saúde, Centro Brasileiro de Análise e Planejamento. Brasília, DF; 2009. 
10. Tonial SR, Silva AAM. Saúde, Nutrição e Mortalidade Infantil no Maranhão. São Luis: Gráfica Universitária UFMA; 1997.

11. Aquino R, Oliveira NF, Barreto ML. Impact of the Family Health Program on Infant Mortality in Brazilian Municipalities. Am J Public Health 2009, 99: 87-93.

12. Olinto MTA, Victora CG, Barros FC, Tomasi E. Determinantes da desnutrição infantil em uma população de baixa renda: um modelo de análise hierarquizada. Cad Saúde Pública 1993; 9(S1): 14-27

13. Alves MTSSB, Sousa SMPS, Coimbra LC. Atenção a Saúde do adulto e da criança no Maranhão. São Luis: EDUUFMA; 2009.

14. ABEP. Critério de Classificação Econômica Brasil 2009. Disponível em www.abep.org.br/mural/abep/cceb. htm.2003 [Acessado em maio de 2009]

15. World Health Organization. Child Growth Standards: Length/height-for-age, weight-forage, weight-for-length, weight-for-height and body mass index-for-age. Methods and development. Geneva, Switzerland: WHO; 2006.

16. Brasil. Vigilância Alimentar e Nutricional SISVAN: Orientações para a coleta e análise de dados antropométricos em Serviços de Saúde. Brasília: Ministério da Saúde; 2008.

17. WHO Anthro. [programa de computador].Versão 3. World Health Organization; 2009

18. Stata Corp. Stata Topics Survey Data Analysis. UCLA: Academic Technology Services, Statistical Consulting Group. Disponível em http://www.ats.ucla.edu/stat/ Stata/topics/ Survey.htm. [Acessado em 14 de abril de 2010]
19. Martorell R, Khan LK, Hughes ML, Grummer-Strawn LM. Obesity in Latin American Women and children. $J$ Nutr 1998; 128: 1464-73.

20. Post CL, Victora CG, Barros FC, Horta BL, Guimarães PRV. Desnutrição e obesidade infantis em duas coortes de base populacional no Sul do Brasil: tendências e diferenciais. Cad Saúde Pública 1996; 12: 49-57.

21. Corso ACT, Botelho LJ, Zeni LAZR, Moreira EAM. Sobrepeso em crianças menores de 6 anos de idade em Florianópolis, SC. Rev Nutr 2003; 16: 21-8.

22. Vitolo MR, Gama CM, Bortolini GA, Campagnolo PDB, Drachler MDL. Alguns fatores associados a excesso de peso, baixa estatura e déficit de peso em menores de 5 anos. J Pediatr 2008; 84: 251-7.

23. Batista Filho M, Souza AI, Miglioli TC, Santos MC. Anemia e obesidade: um paradoxo da transição nutricional brasileira. Cad Saúde Pública 2008; 24: 247 57.

24. Mondini L, Monteiro CA. Relevância epidemiológica da desnutrição e da obesidade em distintas classes sociais: métodos de estudo e aplicação à população brasileira. Rev Bras Epidemiol 1998; 1: 28-39.

25. Lima ALL, Silva ACF, Konno SC, Conde WL, Benicio MHDA, Monteiro CA. Causas do declínio acelerado da desnutrição infantil no Nordeste do Brasil (1986-19962006). Rev Saúde Pública 2010; 44: 17-27.

26. Carvalhaes MABL, Benício MHDA. Capacidade materna de cuidar e desnutrição infantil. Rev Saúde Pública 2002; 36: 188-97.

27. Batista Filho M, Rissin A.. A transição nutricional no Brasil: tendências regionais e temporais. Cad Saúde Pública 2003; 19: 181-91. 